\title{
Effect of a skin-deep surface zone on the formation of a two-dimensional electron gas at a semiconductor surface
}

\author{
Natalia Olszowska, ${ }^{1}$ Jakub Lis, ${ }^{1}$ Piotr Ciochon, ${ }^{1}$ Lukasz Walczak, ${ }^{2}$ Enrique G. Michel, ${ }^{2}$ and Jacek J. Kolodziej ${ }^{1, *}$ \\ ${ }^{1}$ Faculty of Physics, Astronomy, and Applied Computer Science, Jagiellonian University, Łojasiewicza 11, 30-348 Kraków, Poland \\ ${ }^{2}$ Departmento de Física de la Materia Condensada and Condensed Matter Physics Center (IFIMAC), \\ Universidad Autónoma de Madrid, 28049 Madrid, Spain \\ (Received 11 February 2016; revised manuscript received 20 June 2016; published 12 September 2016)
}

\begin{abstract}
Two-dimensional electron gases (2DEGs) at surfaces and interfaces of semiconductors are described straightforwardly with a one-dimensional (1D) self-consistent Poisson-Schrödinger scheme. However, their band energies have not been modeled correctly in this way. Using angle-resolved photoelectron spectroscopy we study the band structures of 2DEGs formed at sulfur-passivated surfaces of InAs(001) as a model system. Electronic properties of these surfaces are tuned by changing the $S$ coverage, while keeping a high-quality interface, free of defects and with a constant doping density. In contrast to earlier studies we show that the Poisson-Schrödinger scheme predicts the 2DEG band energies correctly but it is indispensable to take into account the existence of the physical surface. The surface substantially influences the band energies beyond simple electrostatics, by setting nontrivial boundary conditions for 2DEG wave functions.
\end{abstract}

DOI: 10.1103/PhysRevB.94.115305

\section{INTRODUCTION}

Two-dimensional electron gases (2DEGs) occurring at surfaces of semiconductors have been studied for many years due to their rich phenomenology and extreme technological relevance [1-13]. The 2DEGs arise following subsurface confinement of conduction electrons caused by an electric field. A characteristic quasi-2D surface electronic structure (a set of 2D subbands) is then observed. Depending on the semiconductor doping the phenomenon may take the form of the charge accumulation or the charge inversion layer. A few crystals host native charge accumulation/inversion layers at their surfaces, notably $\mathrm{InAs}, \mathrm{CdO}, \mathrm{InN}, \mathrm{In}_{2} \mathrm{O}_{3}$, and $\mathrm{SrTiO}_{3}$. In many other cases the layers can be intentionally engineered, using adsorbates. Analogous phenomena are found at many interfaces [14-20].

Theoretical modeling of the 2DEGs builds upon the assumption that the surface electrostatic potential is screened by a degenerate electron gas residing in a subsurface potential well. This is formulated as a 1D self-consistent PoissonSchrödinger problem. The problem has been solved iteratively [21-24] and also using the modified Thomas-Fermi approximation (MTFA) [25-28]. These two strategies have been found equivalent $[26,29]$. Nonetheless, angle-resolved photoelectron spectroscopy (ARPES) studies of the 2DEGs band structure have evidenced that the subband energies are not described well by such models [3,6,7]. It has been then proposed that taking into account many-body interactions could resolve the problem [3]. However, these interactions are known to be negligible [23] at least in some of the problematic 2 DEG systems. Thus today, nearly 50 years after the first experimental evidence of 2DEG [14], there is still no complete understanding of the 2DEG systems.

In the present paper we revisit the problem by combining experimental and theoretical studies. Experimental ARPES results and theoretical calculations within the above discussed

\footnotetext{
*jj.kolodziej@uj.edu.pl
}

Poisson-Schrödinger scheme, obtained for an InAs based 2DEG, are brought into harmony after considering adequate boundary conditions for the 2DEG wave functions at the surface. These boundary conditions are traced back to the nontrivial potential interpolation between the crystal and vacuum.

\section{EXPERIMENTAL METHODS}

We focus on InAs, as it is probably the best studied material showing native accumulation layers [1-3,9,10,30-32], having as well a large technical application potential [33-35]. The band bending at InAs surface depends on its orientation and reconstruction as well as on adsorbates $[2,9,10,30,32,36-$ 40]. We use sulfur treatment on the (001) surface in order to control the bending [41].

Our samples are nominally undoped, $n$-type $\operatorname{InAs}(001)$ wafers with a donor concentration of $3 \times 10^{16} \mathrm{~cm}^{-3}$. Sputtering of the surfaces is avoided, as it produces many electrically active defects in the subsurface region [32,42]. The samples are treated with a $\mathrm{HCl}$-iPA solution in $5 \mathrm{~N}$ nitrogen atmosphere, rinsed by iPA, blown dry, and transferred to ultrahigh vacuum without being exposed to atmospheric air, then annealed to prepare In and As terminated surfaces [32,43]. A $S_{2}$ beam is generated using an electrochemical cell $[44,45]$. The $\mathrm{S}$ adsorbed surfaces are annealed in steps to increasingly higher temperature and their reconstructions are monitored using LEED. Most of ARPES studies are done using $\mathrm{He} \mathrm{I} \alpha$ radiation, at $80 \mathrm{~K}$. Variable photon-energy ARPES spectra are acquired at APE beamline at Elettra storage ring in Trieste (Italy).

We study a few differently reconstructed surfaces, prepared with S adsorption on both In and As-terminated InAs(001). Little is known about their atomic structures [39,46-48] but it is not crucial for our discussion, as only values of the total band bending explicitly enter the model under consideration. A very important observation is that, due to the relatively low processing temperatures $[49,50]$, the doping density in the subsurface region remains unchanged for all samples studied 

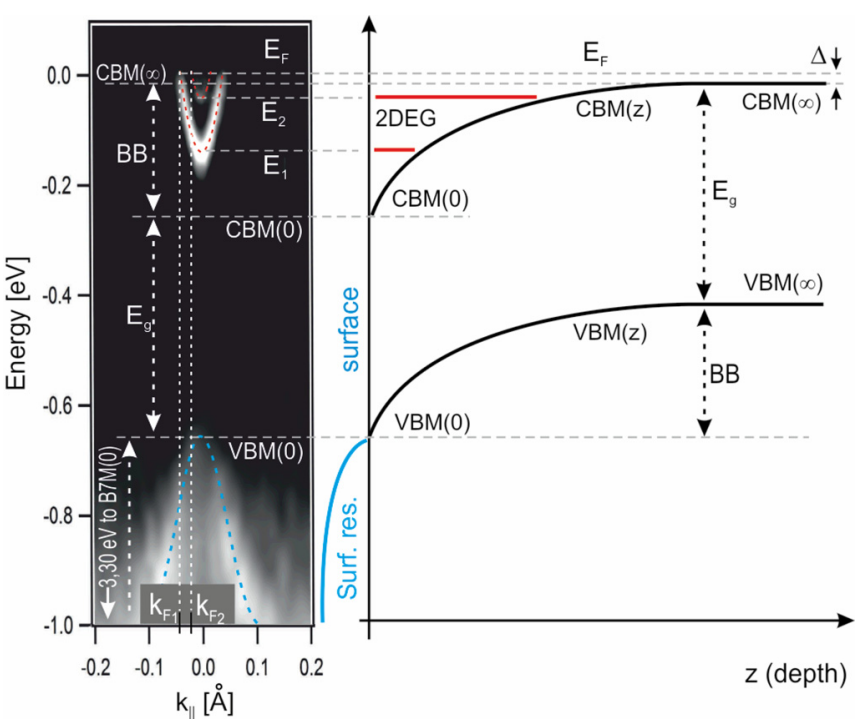

FIG. 1. ARPES image of the electronic structure of exemplary InAs surface including a $2 \mathrm{DEG}$ and the corresponding band diagram. Data acquired using the $\mathrm{He} \mathrm{I} \alpha$ excitation, at $T=80 \mathrm{~K}$.

and equals that of the bulk. Thus unnecessary complications such as uncertain $\Delta$ (see below for the definition) and unbound- charge screening effects are eliminated on the experimental level.

\section{EXPERIMENTAL RESULTS}

The band diagram defining the parameters and conventions used throughout the paper is shown in Fig. 1. $E_{F}$ is the Fermi level for which we assign the zero energy. The bulk electronic structure is represented by a depth dependent valence band maximum $\operatorname{VBM}(z)$ and conduction band minimum $\operatorname{CBM}(z)$. The band gap $\left(E_{g}\right)$ is the difference between the two. At the temperatures relevant to our experiment $(\sim 80 \mathrm{~K}) E_{g}$ is equal to $0.40 \mathrm{eV}$ [51]. The $E_{F}$ to $\mathrm{CBM}(\infty)$ distance is denoted as $\Delta . \Delta$ is calculated using standard formulas [52] and it is small $(\sim 10 \mathrm{meV})$ for our samples. The total band bending is obtained as $\mathrm{BB}=-\operatorname{VBM}(0)-E_{g}-\Delta$. Throughout this paper the variable $z \geqslant 0$ denotes the depth beneath the surface located at $z=0$.

Our study shows that the 2DEG subbands on InAs are not well resolved (as in many earlier reports, see Ref. [53]) if the sample quality is not excellent-see the exemplary spectrum in Fig. 2(a). However, we limit our discussion to examples where the subbands are seen clearly, cf. Figs. 2(b)-2(d). The

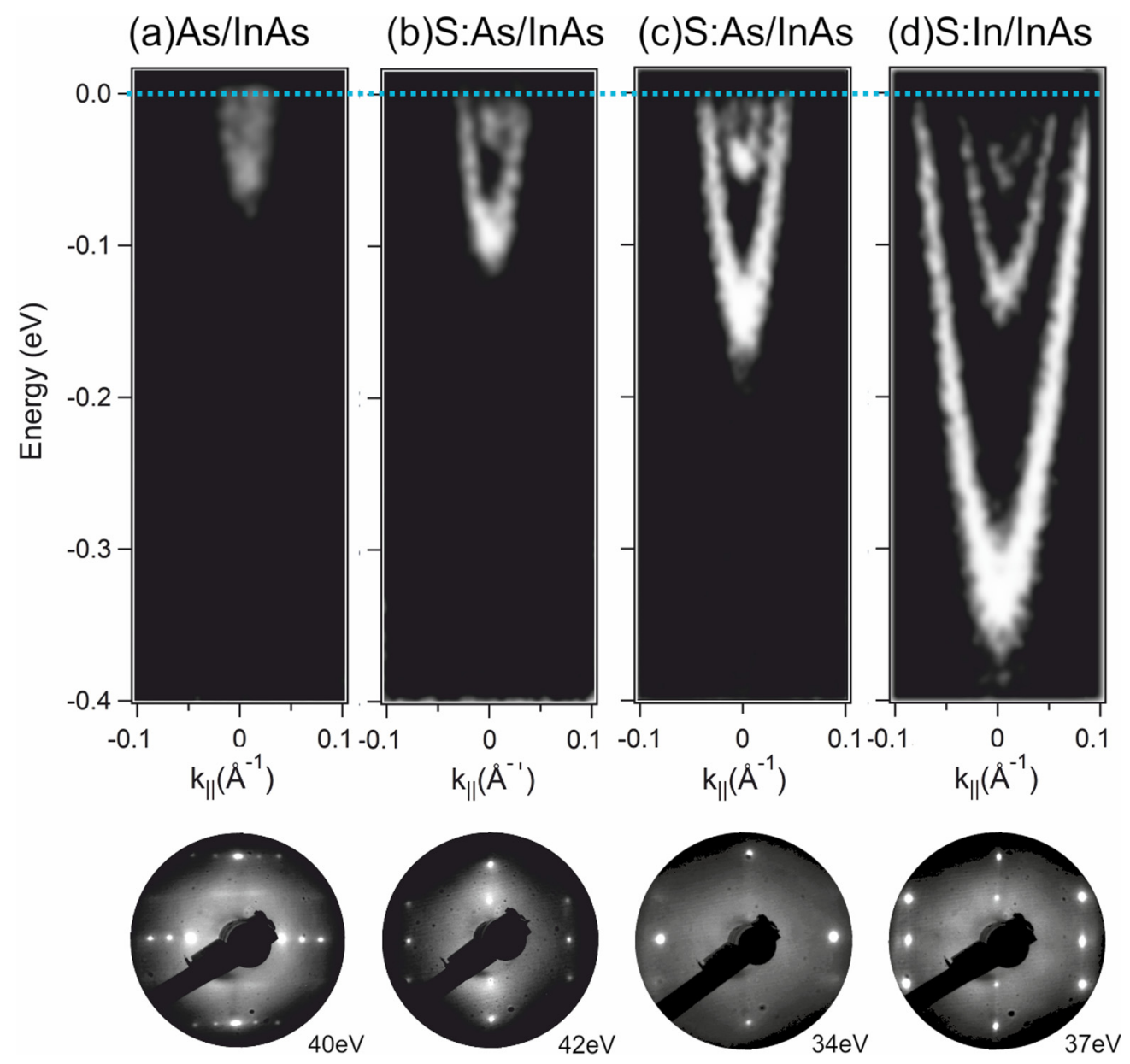

FIG. 2. ARPES photocurrent maps reflecting 2 DEG bands on InAs(001) surfaces, along $\overline{\Gamma J^{\prime}}$ direction: (a) on $c(2 \times 8)-2 \times 4$ As-rich surface, (b) and (c) on sulfur-passivated As-rich $2 \times 1$ and $1 \times 1$ reconstructed surfaces, respectively, and (d) on sulfur-passivated In-rich surface reconstructed $2 \times 1$. LEEDs for the surfaces are shown in the lower row. Data acquired using the He I $\alpha$ excitation, at $T=80 \mathrm{~K}$. 
(a) $\ln / \ln A s$

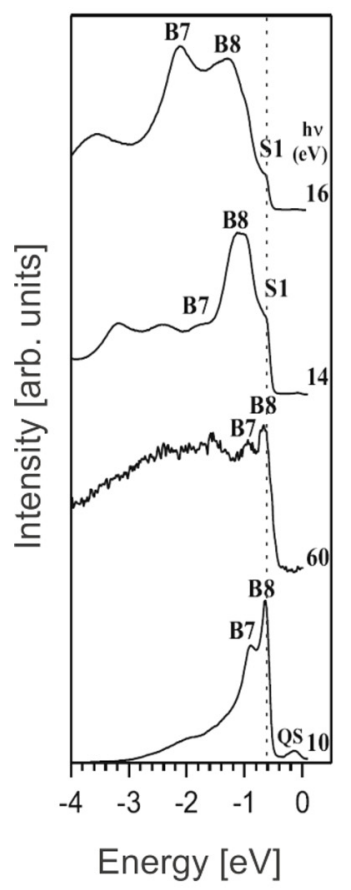

(b) $\ln / \ln A s$

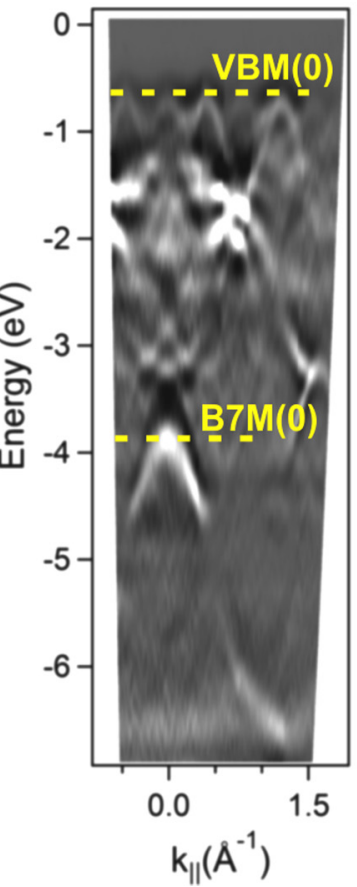

(c) As/lnAs

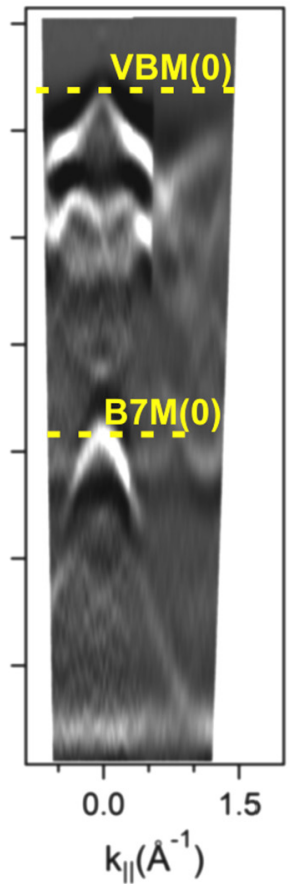

(d) S:As/InAs

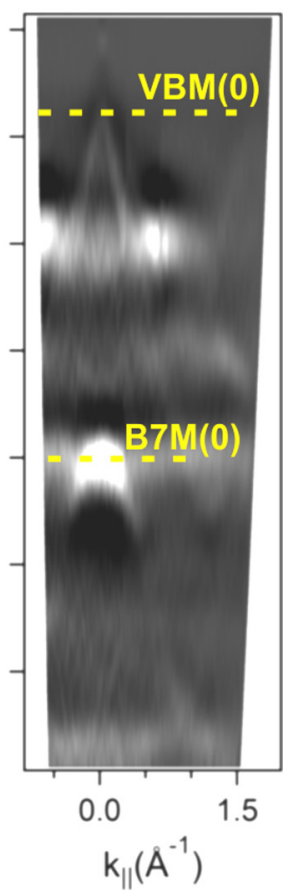

FIG. 3. (a) Valence band of In-rich $c(8 \times 2)-4 \times 2 \operatorname{InAs}(001)$ investigated using variable photon energy ARPES at normal emission (second derivative of the photocurrent). Bulk (B7, B8) and surface (S1) related features are indicated. QS corresponds to emission from the 2DEG. (b)-(d) Valence band structures along $\overline{\Gamma J^{\prime}}$ direction imaged with ARPES: correspondingly for $c(8 \times 2)-4 \times 2$ In-rich surface, $c(2 \times 8)-2 \times 4$ As-rich surface, and $1 \times 1 \mathrm{~S}$ passivated As-rich surface. Lines denoting $\mathrm{VBM}(0)$ are drawn $3.30 \mathrm{eV}$ above the lines denoting $\mathrm{B} 7 \mathrm{M}(0)$. The $\mathrm{B} 7 \mathrm{M}(0)$ level has been determined by fitting Gaussian line shapes to not differentiated energy spectra. Data acquired using the He I $\alpha$ excitation [except (a)], at $T=80 \mathrm{~K}$.

subbands are found isotropic, so there is no need to consider their azimuthal orientation.

In order to determine the $\mathrm{BB}$ value we notice that the VBM in InAs corresponds to the $\Gamma_{8}$ level, which is the top of the B8 bulk band [54]. When measured with normal emission, variable excitation energy ARPES, the B8 band reaches maximal value at $10 \mathrm{eV}$ photon energy and again at $60 \mathrm{eV}$ [see Fig. 3(a) and Appendix A], meaning that the bulk $\Gamma$ point is probed for these energies. We also notice that, for the In-terminated (001)InAs surface, there exists a nondispersing surface resonance (S1), clearly seen at photon energies of 14 and $16 \mathrm{eV}$, when it is separated from bulk bands. As seen in Fig. 3(a) $\operatorname{VBM}(0)$ and $\mathrm{S} 1$ coincide (at $-0.55 \mathrm{eV}$ ). Thus, for the In-terminated (001)InAs surface one may also find the $\operatorname{VBM}(0)$ simply by studying the onset of the valence band. In order to determine the BB using He I $\alpha$ excited ARPES for other samples we find the surface independent reference, that is the apparent B7 band maximum [B7M(0)], see Fig. 3(b). Based on the detailed studies of the clean In-rich InAs surface we find the difference in energy between the $\operatorname{VBM}(0)$ and $\mathrm{B} 7 \mathrm{M}(0)$ as $3.30 \mathrm{eV}$. Then the $\mathrm{BB}$ values are found using the formula $\mathrm{BB}=-\mathrm{B} 7 \mathrm{M}(0)-3.30 \mathrm{eV}-E_{g}-\Delta$ [see also Figs. 3(c) and 3(d)]. As seen in Fig. 3, surface resonances are present at the $\bar{\Gamma}$ point, aligned with the $\operatorname{VBM}(0)$ to within accuracy of our measurements, both for clean and S-passivated InAs(001) surfaces. Similar results have been obtained before [28,32,55].

Based on the spectra shown in Fig. 2 one may extract a few numerical results including the energies corresponding to the subband minima $\left(E_{i}\right)$ and Fermi wave vectors for the subbands $\left(k_{F_{i}}\right)$, see also Fig. 1 for explanation. These results are given in Table I. Earlier studies (see for example Ref. [3]) have revealed that MTFA underestimates the binding energies corresponding to subband minima $E_{i}$. Our experimental data are similarly incoherent with the energy spectra calculated from the MTFA, see the case $\lambda \rightarrow \infty$ [i.e., $\arctan (\lambda)>1$ ] in Fig. 4.

TABLE I. Measured 2DEG properties for clean and S passivated InAs surfaces. See Fig. 1 for definitions of the parameters shown. The sheet electron density $n_{2 \mathrm{D}}$ is estimated by the Luttinger area: $n_{2 \mathrm{D}}=\sum_{i} k_{F_{i}}^{2} /(2 \pi)$. Uncertainties are: $50 \mathrm{meV}$ for $\operatorname{VBM}(0)$ and $\mathrm{BB}$, $5 \mathrm{meV}$ for $\mathrm{E}_{i}$, and $0.005 \AA^{-1}$ for $k_{F_{i}}$.

\begin{tabular}{lccccccc}
\hline \hline & \multicolumn{2}{c}{ On the In-rich surf. } & & \multicolumn{2}{c}{ On the As-rich surface } \\
\cline { 2 - 3 } \cline { 6 - 7 } & Clean & $2 \times 1(S)$ & & Clean & $1 \times 1(S)$ & $2 \times 1(S)$ \\
\hline $\operatorname{VBM}(0)(\mathrm{eV})$ & -0.55 & -0.98 & & -0.52 & -0.69 & -0.62 \\
$\mathrm{BB}(\mathrm{eV})$ & 0.15 & 0.58 & & 0.12 & 0.29 & 0.22 \\
$E_{1}(\mathrm{eV})$ & - & -0.33 & & - & -0.15 & -0.09 \\
$E_{2}(\mathrm{eV})$ & - & -0.13 & & - & -0.04 & -0.02 \\
$E_{3}(\mathrm{eV})$ & - & -0.05 & & - & - & - \\
$k_{F_{1}}\left(\AA^{-1}\right)$ & - & 0.090 & & - & 0.040 & 0.030 \\
$k_{F_{2}}\left(\AA^{-1}\right)$ & - & 0.050 & & - & 0.015 & 0.010 \\
$k_{F_{3}}\left(\AA^{-1}\right)$ & - & 0.015 & & - & - & - \\
$n_{2 \mathrm{D}}\left(10^{12} \mathrm{~cm}^{-2}\right)$ & - & $17(2)$ & & - & $2.9(7)$ & $1.6(5)$ \\
\hline \hline
\end{tabular}




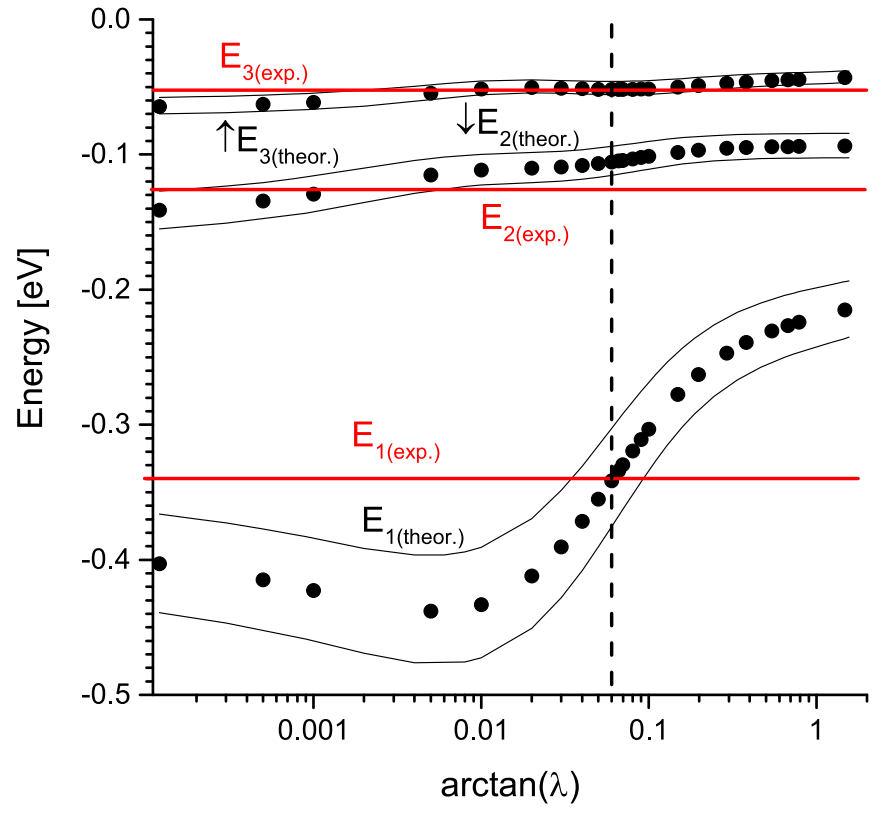

FIG. 4. Calculated dependencies of the theoretical 2DEG subband minima on the parameter $\lambda$ (points), for the case of $\mathrm{BB}=$ $0.58 \mathrm{eV}$. Thin solid lines show the binding energies for $\mathrm{BB} \pm$ $50 \mathrm{meV}$, which reflects the experimental uncertainty. Corresponding experimental energies are indicated by horizontal lines (red online). For $\arctan (\lambda)>1$ MTFA solutions are reached. The vertical line indicates the $\lambda$ value for which the theoretical and experimental binding energies $E_{i}$ match the most closely.

\section{THEORETICAL FRAMEWORK}

In order to explain such discrepancies King et al. [3] proposed that many-body interactions within the accumulation layers (neglected when using MTFA) cause a giant renormalization of the InAs band gap down to $0.1 \mathrm{eV}$, at the surface. We find this statement questionable. It is well known that in narrow direct-gap III-V semiconductors, for typical electron densities found in accumulation layers $\left(10^{18}-10^{19} / \mathrm{cm}^{3}\right)$, the many-body interactions do not impact the band gap substantially. This follows from the fundamental theory of the electron gas, see Ref. [23]. These theoretical expectations are soundly confirmed in experiments - the band gap renormalization is measured for degeneratively $n$-doped GaAs in several studies-see Ref. [56] and references therein. The renormalization is found not to exceed $100 \mathrm{meV}$, for doping concentrations $10^{18}-10^{19} / \mathrm{cm}^{3}$. This is scaled with the factor $\sim 1 / 2$ to represent InAs [57] but more than $3 / 4$ of the effect is due to interactions of conduction electrons with ionized donors [58], nonexistent for the 2DEG case. Thus, the many-electron interactions, for the relevant densities, reduce the InAs band gap only by $\sim 10 \mathrm{meV}$. While there are indications that the discussed effects increase when going from $3 \mathrm{D}$ to $2 \mathrm{D}$ systems they most likely remain not relevant for the InAs 2DEG band structure description in the first order [59].

Having said this, we expect the one-electron PoissonSchrödinger calculation to be applicable to InAs. We follow the calculation scheme outlined in Ref. [28] using the effective mass and envelope wave function approximation but we lift specific surface boundary conditions imposed by MTFA. We
TABLE II. Evaluated energies of the subbands minima for 2DEG corresponding to the experimentally investigated band bending and related values of $\lambda$. Additionally solutions corresponding to $\lambda \rightarrow \infty$ are shown with the smaller numbers.

\begin{tabular}{lccc}
\hline \hline $\mathrm{BB}$ & $0.58 \mathrm{eV}$ & $0.29 \mathrm{eV}$ & $0.22 \mathrm{eV}$ \\
\hline$E_{1}(\mathrm{eV})$ & $-0.34 /-0.20$ & $-0.15 /-0.10$ & $-0.09 /-0.08$ \\
$E_{2}(\mathrm{eV})$ & $-0.11 /-0.09$ & $-0.045 /-0.04$ & $-0.03 /-0.03$ \\
$E_{3}(\mathrm{eV})$ & $-0.05 /-0.04$ & & \\
$\lambda\left(\AA^{-1}\right)$ & $0.06 / \infty$ & $0.05 / \infty$ & $0.09 / \infty$ \\
\hline \hline
\end{tabular}

discuss the calculation procedure in Appendix B in more detail. There are two steps in the calculations. First, the electrostatic potential $V$ is calculated within the band bending approximation from the one-dimensional Poisson equation. Second, the one-dimensional Schrödinger equation is solved (the separated solutions in the dimensions parallel to the surface are left intact)

$$
\left[-\frac{\hbar^{2}}{2 m_{\mathrm{cb}}} \frac{d^{2}}{d z^{2}}+V(z)\right] \psi=E \psi
$$

where $m_{\mathrm{cb}}$ stands for the effective mass in the conduction band. In general, the above equation on the semiaxis corresponds to a Hermitian (self-adjoint) operator if it acts on functions $\xi$ satisfying the following boundary condition at the origin [61]:

$$
\frac{d}{d z} \xi(0)=\lambda \xi(0)
$$

where $\lambda$ has dimension of inverse length and can have any value including infinity limit. We restrict our consideration to $\lambda>0$, see Appendix $\mathrm{C}$ for a justification. So far only the particular case with $\lambda \rightarrow \infty$ corresponding to $\xi(0)=0$ has been considered $[27,28]$, resulting in the basis of generalized wave functions (in the direction normal to the surface) $\sin (k z)_{k>0}$.

We treat $\lambda$ as a parameter to be fit to the data. As shown in Fig. 4, the subband energies $E_{i}$ depend on $\lambda$ significantly and it is possible to nearly match the calculated and the experimental energies $E_{i}$ by selecting $\lambda$. Complete sets of energies $E_{i}$ evaluated within this scheme are shown in Table II. They are close to the experimental values given in Table I.

\section{DISCUSSION}

Figures 5(a)-5(d) schematically illustrates how $\lambda$ impacts ground-state solutions $\psi$. For $\lambda=0$ the maximum of the wave function is located at the origin, corresponding to a large negative potential energy $\langle\psi|V| \psi\rangle$. As $\lambda$ grows, the maximum appears at some distance from $z=0$, decreasing the binding energies of the 2DEG electrons. Another important factor is the kinetic energy, which measures the variation of the wave function amplitude and hence it is larger for $\lambda=\infty$ than for $\lambda=0$. The values of $\lambda$ given in Table II are relatively small and the resulting wave function amplitudes at the surface are significant. In agreement with this observation we have shown recently, using ARPES [32], that on a clean InAs(001) surface, 2DEG states acquire the periodicity of the surface reconstruction. This is an experimental evidence that the 2DEG wave function amplitudes may be large at the very surface. In 
(a)

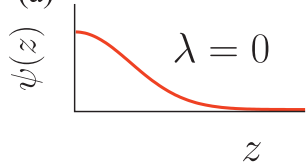

(c)

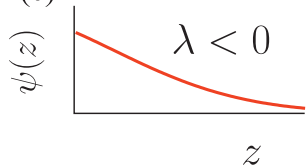

(e) $\quad 0 \quad 20 \quad 40 \quad 60$

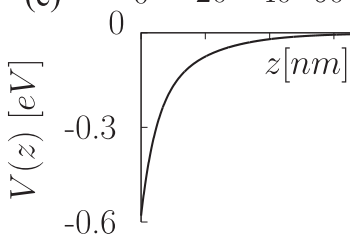

(b)

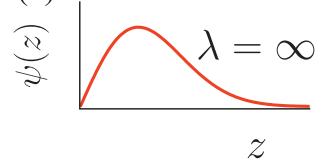

(d)

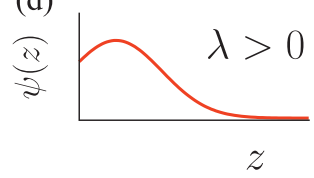

(f)

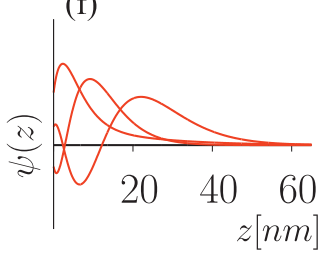

FIG. 5. (a)-(d) Schematic wave functions of the bound state for different values of $\lambda$, as indicated. (e) A potential curve for the case when $\mathrm{BB}=0.58 \mathrm{eV}$. (f) Wave functions corresponding to eigenenergies: $-0.34,-0.11$, and $-0.05 \mathrm{eV}$, for $\mathrm{BB}=0.58 \mathrm{eV}$ and $\lambda=0.06$.

order to illustrate the characteristic length scale concerning the investigated problem an exemplary potential curve and corresponding wave functions are shown in Figs. 5(e) and 5(f).

Relation (2) is in fact a generalization of the so-far considered model. To see this, we write the basis of generalized eigenfunctions as $\cos [k z+\phi(k)]$. The phase factor $\phi$ satisfies

$$
\cos [\phi(k)]=-\operatorname{sgn}(\lambda) \frac{k}{\sqrt{k^{2}+\lambda^{2}}} .
$$

For small wave vectors $(k \rightarrow 0)$ the phase $\phi$ approaches $-\pi / 2$, so $\cos [k z+\phi(k)] \approx \sin (k z) ; \lambda=0$ is the only exception from the rule. It is also interesting to note that for shallow subbands, located less than $0.1 \mathrm{eV}$ below $E_{F}$, corrections to their minimal energies resulting from allowing different values of $\lambda$ are not large, see Table II. Therefore our results agree with earlier calculations by Abe et al. [62], referring to such shallow subbands, despite the fact that the specific surface boundary condition, corresponding here to $\lambda \rightarrow \infty$, has been only considered by Abe et al.

In the envelope wave function approximation the trigonometric functions on the semiaxis can be regarded as coming from the interference between the incoming and outgoing waves

$$
\frac{1}{2}\left(e^{i k z}+e^{-i k z+2 i \phi}\right)=e^{i \phi} \cos (k z-\phi),
$$

with the reflection coefficient equal to unity. The phase $\phi$ is the only remnant of the reflection caused by a nontrivial potential far from the surface. The condition used so far is equivalent to $\phi= \pm \pi$ and its rationale is given in the literature: "As the characteristic penetration length of the wave functions into the vacuum is very short, much shorter than the variation of carrier density in the space-charge region, it is a good approximation to impose the boundary condition that the wave function is equal to zero at the surface and thus that the carrier concentration tends smoothly to zero at the surface" [28]. No doubt, the

electron wave function dies off outside the crystal. But, this does not mean that the the generalized wave functions have to be $\sin (\cdot)$ functions. This is true only if the surface is modeled as a featureless, infinite potential step, see Appendix C.

The envelope wave function is an effective description of phenomena on large scales compared to the lattice constant. Thus, there is no unambiguous method to put the surface plane into the system. Furthermore, there is an about $1 \mathrm{~nm}$ wide zone at the surface corresponding to the reconstructed layers. In this zone the assumptions of the envelope approximation do not hold. So, the realistic model of 2DEG should include three regions: the vacuum region with negligible wave function density, the crystal bulk where free-electron approximation works well, and a transition zone where the potential interpolates between its vacuum and bulk values. We solve the electrostatic problem in the crystal bulk leaving $\lambda$ as an effective parameter accounting for the transition zone properties. In Appendix C we discuss simple models illustrating these ideas.

\section{CONCLUSIONS}

We have presented coherent experimental and theoretical studies on 2DEG band structure for a few differently reconstructed InAs(001) surfaces, treated as a model system. The surfaces has been carefully chosen to avoid experimental problems that are often present for the kind of the spectroscopic studies done. Surface band bending values have been cautiously evaluated. Thus, the obtained experimental material provides a robust test of the applied theoretical concepts. We find that a simple one-electron Poisson-Schrödinger model explains the 2DEG band energies well, provided that the surface model is not oversimplified, i.e., proper boundary conditions on the 2DEG wave functions are imposed. We show that the conditions effectively describe the "skin-deep surface zone" (or the physical surface) and correspond to a nontrivial surface potential. Neglecting the "skin effect," what has been overlooked so far, leads to heavily underestimated 2DEG band energies. For InAs we find this effect solely being able to reconcile the measured and calculated energy spectra. Therefore we think that many-body corrections may be not taken into account to first order, in the context of surface 2DEG in InAs, but they may be more pronounced for other materials. The skin effect is, in principle, present for any $2 \mathrm{DEG}$ system, so that our findings impact also the understanding and modeling of two-dimensional electron gases existing at surfaces and interfaces of other semiconductors and oxides. Thus, further work is required to understand rigorously all aspects of the 2DEG band structure.

\section{ACKNOWLEDGMENTS}

We acknowledge financial support by Polish NCN (Contract 2011/03/B/ST3/02070). The research was carried out with the equipment purchased thanks to European Regional Development Fund in the framework of the Polish Innovation Economy Operational Program (Contract No. POIG.02.01.0012-023/08). L.W. and E.G.M. acknowledge financial support by MINECO (Grant MAT2014-52477-C5-5-P). J.L. wishes to thank Professor K. Rościszewski for instructive discussion. 

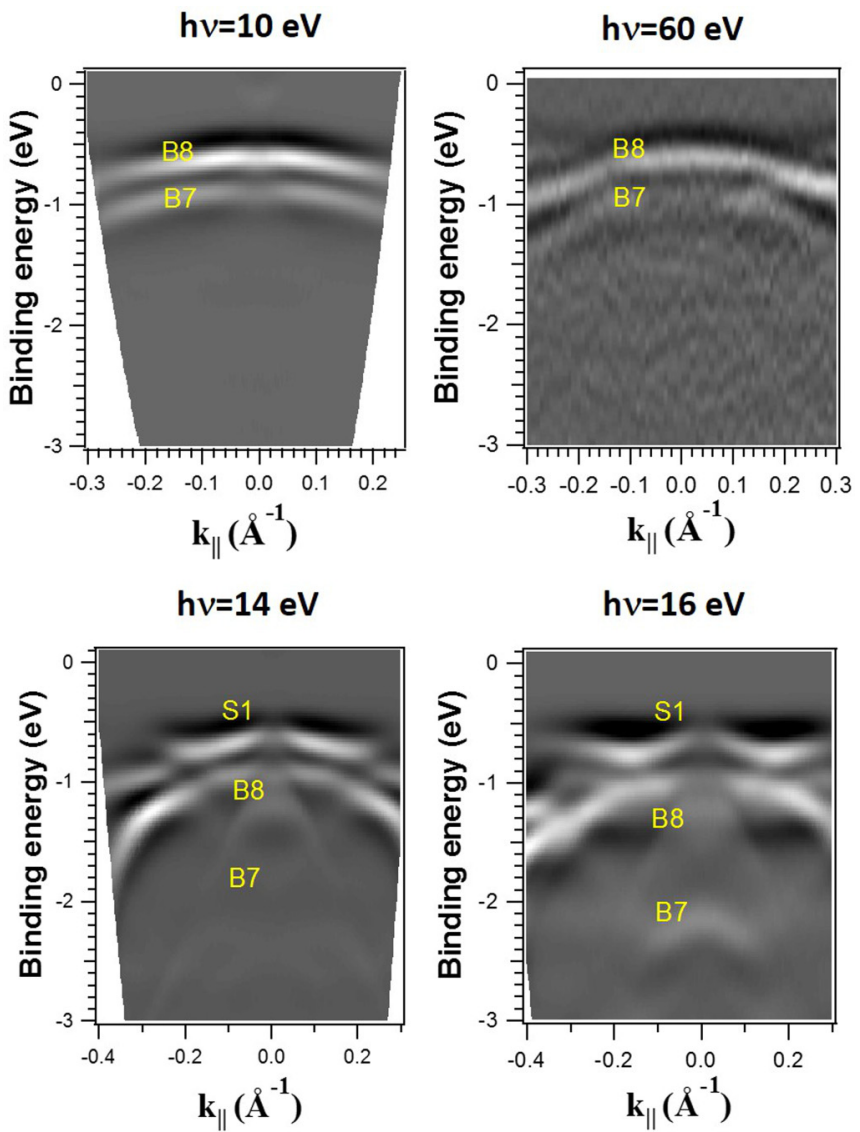

FIG. 6. ARPES photocurrent maps (second derivative) along $\Gamma J^{\prime}$ for 10 and $60 \mathrm{eV}$ (top) and for 14 and $16 \mathrm{eV}$ (bottom) photon energy. The maps are collected for the InAs crystal terminated with clean In-rich (001) surface, reconstructed $c(8 \times 2)-4 \times 2$. Energy is measured relative to the Fermi level.

\section{APPENDIX A: DETERMINATION OF VBM(0)}

This Appendix is intended to assist the interpretation of the spectra shown in Fig. 3(a). Four parts of Fig. 6 show angle-resolved photocurrent maps measured using different photon energies. The two top maps correspond to 10 and 60 $\mathrm{eV}$ photon energies for which the bulk $\Gamma$ point is probed. For these maps the valence band region is dominated by bulk bands B8 and B7 and the VBM may be directly read as the energy of the $\mathrm{B} 8$ band at $k_{\|}=0$. In contrast, the two bottom maps correspond to 14 and $16 \mathrm{eV}$ photon energies and illustrate the situation when the B8 band is seen away from the VBM. In these cases the valence band region is dominated by surface resonances, including the indicated S1 coinciding, at $k_{\|}=0$, with the VBM.

\section{APPENDIX B: CALCULATION SCHEME}

Here we outline the calculation procedure in detail. As the numerical procedures involved are not recourse demanding we take into account the following bands: light holes, heavy holes, conduction band, and donor density (this is done for the universality of the model while for the investigated samples the hole bands as well as the donor density factor could be neglected). The conduction band minimum corresponds to the zero energy. The charge density accumulated in the conduction band reads

$$
n_{\mathrm{cb}}=\frac{1}{\pi^{2}} \int_{0}^{\infty} \frac{k^{2} d k}{1+\exp \beta\left[E_{\mathrm{cb}}\left(k^{2}\right)-E_{F}+V(z)\right]},
$$

where $\beta=1 / k_{B} T$ with $k_{B}$ denoting the Boltzmann constant, $T$ is the temperature, and $V(z)$ is the electrostatic potential multiplied by the electron charge. We use the $\mathbf{k} \cdot \mathbf{p}$ relation for the kinetic energy of the electrons

$$
E_{\mathrm{cb}}\left(k^{2}\right)=\frac{\hbar^{2}}{2 m_{e}} k^{2}+\frac{E_{g}}{2}\left(\sqrt{1+4 P^{2} k^{2}}-1\right)
$$

where $m_{e}$ stands for the free electron mass. $E_{g}$ is calculated from the Varshni parametrization [28]

$$
E_{g}=0.415-\frac{2.76 \times 10^{-4} T^{2}}{83+T}
$$

where $T$ is given in Kelvin and the energy in $\mathrm{eV}$, and

$$
P^{2}=\frac{3 \hbar^{2}}{2 m_{e}}\left(\frac{m_{e}}{m_{c b}}-1\right) \frac{E_{g}+\delta}{\left(3 E_{g}+2 \delta\right) E_{g}} .
$$

$\delta$ stands for the spin-orbit coupling, here $\delta=0.381 \mathrm{eV} . m_{\mathrm{cb}}=$ $0.024 m_{e}$ denotes the electron effective mass in the conduction band. The formula for hole density reads

$$
p_{i}=\frac{1}{\pi^{2}} \int_{0}^{\infty} \frac{k^{2} d k}{1+\exp \beta\left(E_{g}+\frac{\hbar^{2}}{2 m_{i}} k^{2}+E_{F}-V(z)\right)},
$$

where $i \in\{l h, h h\}$ stands for light and heavy holes, respectively. The numerical values of the hole masses are $0.021 m_{e}$ and $0.41 m_{e}$, respectively. The density of (positively) ionized donors in nondegenerate semiconductors reads

$$
\tilde{p}_{d}=\frac{N_{B}}{1+2 \exp \beta\left[E_{F}+E_{B}-V(z)\right]},
$$

where the considered donor density is $N_{B}=3 \times 10^{16} \mathrm{~cm}^{-3}$ and the energy of shallow donors is denoted with $E_{B}$. However, the average distance between the doping atoms is $N_{B}^{-1 / 3}=32$ $\mathrm{nm}$, while the Bohr radius of the hydrogenic shallow donor states yields $33 \mathrm{~nm}$. So, at this density the material is rather a poor metal [60], and a full donor ionization should be assumed,

$$
p_{d}=N_{B} .
$$

The electron neutrality condition for $V=0$,

$$
p_{l h}+p_{h h}+p_{d}-n_{\mathrm{cb}}=0,
$$

sets the Fermi level. It is found $14 \mathrm{meV}$ above the conduction band minimum.

Having set the Fermi energy, we can solve the Poisson equation

$$
\frac{d^{2}}{d z^{2}} V(z)=\frac{e_{0}^{2}}{\varepsilon_{0} \varepsilon_{b}}\left[p_{h h}(z)+p_{l h}(z)+p_{d}(z)-n_{\mathrm{cb}}(z)\right],
$$

where $\varepsilon_{0}$ and $\varepsilon_{b}$ stand for the vacuum dielectric constant and InAs static dielectric constant, respectively, and $e_{0}$ for the electron charge. We require that $V(0)$ be equal to the measured band bending and that $V(z)$ vanish in the bulk $(z \rightarrow \infty)$. Hence, the derivative of $V(z)$ at the surface, which 
is interpreted as the surface charge, is not a free parameter. Now we can solve the Schrödinger equation:

$$
\left[E_{\mathrm{cb}}\left(-\frac{d^{2}}{d z^{2}}\right)+V(z)\right] \psi=E \psi,
$$

where $E_{\mathrm{cb}}\left(-\frac{d^{2}}{d z^{2}}\right)$ accounts for the nonparabolic dispersion relation. Various boundary conditions are assumed, as discussed in Sec. IV. Note that in Sec. IV we discussed the parabolic dispersion relation while the actual calculations are performed for nonparabolic $E_{\mathrm{cb}}(k)$. The rationale is that the theory of Hamiltonian operators and related self-adjoint extensions on the semiaxis has been formulated for the Laplace operator. The status of the nonparabolic operators is not clear and our arguments lose their mathematical rigor. However, they seem to be physically reasonable as discussed in Sec. V and below.

The above described scheme is not fully self-consistent. When calculating potential (B9), the plane wave approximation is assumed. This potential is subsequently used to pick out the correct boundary condition. Numerical investigations showed that the choice of boundary condition is not decisive for the potential. It is $\frac{d}{d z} V(0)$ which is sensitive to $\lambda$, making us reluctant to attribute to it the strict physical meaning of the surface charge density.

\section{APPENDIX C: TENTATIVE INTERPRETATION OF $\lambda$}

We argue above that the nontrivial boundary condition imposed on the wave functions are due to the thin interpolation zone between the crystal and vacuum. To be more specific, we discuss below three simple models and show how these intuitions can be quantified. Here, to avoid technical complications, we consider the parabolic dispersion. We begin with the well known step potential

$$
\Theta(z)= \begin{cases}\frac{\hbar^{2}}{2 m} \Theta_{0}, & z<0, \\ 0, & 0>z,\end{cases}
$$

with $\Theta_{0}>0$. The Schrödinger equation has the form

$$
\left(-\frac{\hbar^{2}}{2 m} \frac{d^{2}}{d z^{2}}+\Theta(z)-\frac{\hbar^{2}}{2 m} k^{2}\right) \psi(z)=0,
$$

where $\frac{\hbar^{2}}{2 m} k^{2}$ denotes the energy. The solutions read

$$
\psi(z)= \begin{cases}{\left[\sin \phi_{1}(k)\right] \exp \sqrt{\Theta_{0}-k^{2}} z,} & z<0, \\ \sin \left[k z+\phi_{1}(k)\right], & z>0 .\end{cases}
$$

The phase

$$
\tan \phi_{1}(k)=\frac{k}{\sqrt{\Theta_{0}-k^{2}}}
$$

ensures equality of the derivatives calculated at $0^{+}$and $0^{-}$. For small $k$ the above equation simplifies to

$$
\phi_{1}(k)=\frac{k}{\sqrt{\Theta_{0}}} .
$$

The nontrivial phase appears in the case of the step potential, it is negligible if $\Theta_{0} \gg k^{2}$, so it holds only in the case of the infinite barrier. The interpolating zone can be introduced by an additional step

$$
\Theta(z)=\left\{\begin{array}{lr}
\infty, & z<0, \\
\frac{\hbar^{2}}{2 m} U, & 0<z<z_{0}, \\
0, & z>z_{0} .
\end{array}\right.
$$

We solve the Schrödinger equation (C2) for $0<k^{2}<U$ with the boundary condition $\psi(0)=0$. The solutions read

$$
\psi_{+}(k ; z)=\left\{\begin{array}{cc}
a_{+} \sinh \left(\sqrt{U-k^{2}} z\right), & 0<z<z_{0}, \\
\cos \left(k z+\phi_{+}\right), & z>z_{0},
\end{array}\right.
$$

where $a_{+}$and $\phi_{+}$are parameters to be determined. The continuity of $\psi_{+}$and its first derivative at $z_{0}$ result in the following relation for $\phi_{+}$:

$$
\sqrt{U-k^{2}} \operatorname{coth}\left(\sqrt{U-k^{2}} z_{0}\right)=-k \tan \left(k z_{0}+\phi_{+}\right),
$$

leading for small $k$ to the following formula:

$$
\cos \phi_{+}=-\frac{k}{\sqrt{k^{2}+k_{+}^{2}}},
$$

with

$$
k_{+}=\sqrt{U} \operatorname{coth} \sqrt{U} z_{0}
$$

and

$$
a_{+}=-\frac{k}{\sqrt{U} \cosh \sqrt{U} z_{0}} .
$$

Equation (C9) coincides with Eq. (3) upon identification $\lambda=k_{+}$which allows drawing an analogy between lowenergetic scattering from a nontrivial potential and the abstract condition (2) for $\lambda>0$. The approximate value of the solutions is evident - the resulting wave functions have a common part $\sinh \sqrt{U} z$ on the distance $0<z_{0}$. The matching condition is an approximate one and so are the resulting wave functions. But only this assumption allows switching to the well-defined but simpler Hamiltonian. To this end we consider the potential

$$
V(z)=\left\{\begin{array}{lr}
\infty, & z<0, \\
\frac{\hbar^{2}}{2 m} U+v(z), & 0<z<z_{0}, \\
v(z), & z>z_{0} .
\end{array}\right.
$$

The potential $v(z)$ varies slowly on distances much larger than $z_{0}$. Additionally, we consider $U \gg v(z)$ for $\left(0, z_{0}\right)$, so that the solutions (C7) are valid for $z<z_{0}$. Then we arrive at the equation

$$
\left(-\frac{\hbar^{2}}{2 m} \frac{d^{2}}{d z^{2}}+v(z)-\frac{\hbar^{2}}{2 m} k^{2}\right) \psi(z)=0
$$

for $z>z_{0}$ and with the boundary condition $\psi^{\prime}\left(z_{0}\right)=k_{+} \psi\left(z_{0}\right)$. As a consequence, we obtain an approximate spectrum of the problem (C12), whose quality depends on the mutual relations between $U$ and $v$. Such a separation of problems for $z<z_{0}$ and $z>z_{0}$ is possible in the low energy region only.

Similarly, one can treat the case with $U<0$. Then both $\lambda>0$ and $\lambda<0$ can appear. The latter case corresponds to the model with short-ranged potentials deep enough to produce a single bound state [61].

We limit our discussion to the low-energy sector. This is meaningful, if $k^{2} \ll U$, i.e., the energy of the wave is much 


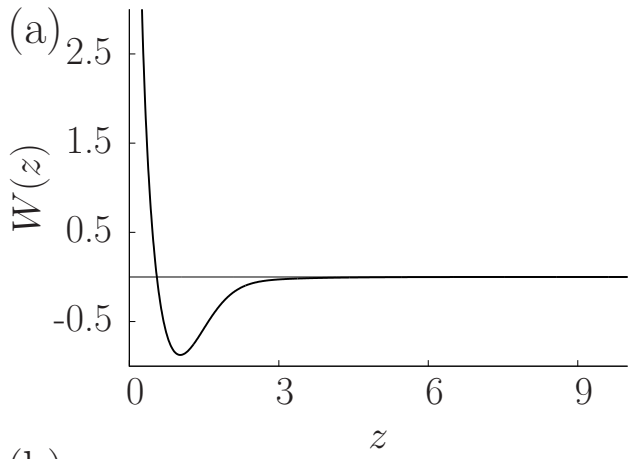

(b)

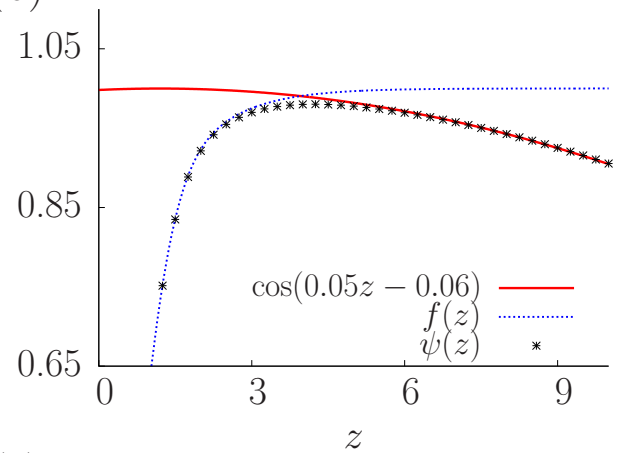

(c)

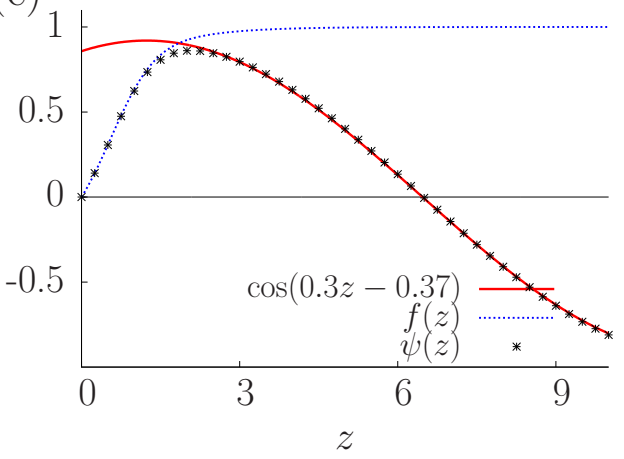

FIG. 7. The threshold potential described in the text (A). Numerical solutions $\psi(z)$ for (B) $k=0.05$, and (C) $k=0.3$ compared with fitted trigonometric functions. The smaller $k$, the smaller the resulting phase according to general arguments given in the text. Note that the smaller $k$ is considered, the longer the solutions $\psi$ stay close to function $f$. For the linearity of the Schrödinger equation we do not give amplitudes of the fitted functions. lower than the barrier height. In physical terms, the barrier corresponds to the material work function $(4-5 \mathrm{eV})$ which is at least ten times more than energies encountered in accumulation layers. There is also a term $k z_{0}$ in $(\mathrm{C} 8)$ which is neglected to arrive at Eqs. (C9)-(C11). As such, the approximation works well if the surface potential range is small compared to the wave vectors $k$.

The step functions are nonphysical. Now we will consider a model with a smooth potential $W$ exploding to infinity for $z=$ 0 . The model has an interesting property: the wave functions vanish at the origin and, nevertheless, they are very close to $\cos (\cdot)$ functions in the region where $W$ is negligible. As such, the parameter $\lambda$ considered above and the behavior of the wave functions at the very surface are not correlated. We consider function $f$,

$$
f(z)=1-\frac{1}{2}\left(e^{-z}+e^{-z^{2}}\right),
$$

which linearly approaches zero at the origin $[f(z) \sim z / 2$ for $z \rightarrow 0]$ and tends to a nonzero value for large $z[f(z) \rightarrow 1$ for $z \rightarrow \infty$ ]. Using this function we define the potential

$$
W(z)=\frac{\hbar^{2}}{2 m f(z)} \frac{d^{2} f(z)}{d^{2} z}
$$

that explodes close to the origin $\left[W(z) \sim+z^{-1}\right.$ for $\left.z \rightarrow 0\right]$ and quickly vanishes for large $z$, see Fig. 7. Trivially, by plugging $k=0$ and $\psi=f$ into Schrödinger equation

$$
\left[-\frac{\hbar^{2}}{2 m} \frac{d^{2}}{d z^{2}}+W(z)-\frac{\hbar^{2}}{2 m} k^{2}\right] \psi(z)=0
$$

one can check that $f$ is a bound, zero-energy solution. It is called zero or threshold resonance [63]. The relevance of $f$ lies in the fact that solutions with the same boundary condition $[\psi(0)=0]$ approach (pointwise) $f(z)$ in the limit $k \rightarrow 0$ [64]. So, the low energy solutions are nearly constant in the region where $f$ is constant and, in this region, appear as $\cos (\cdot)$ functions, see Fig. 7 for a schematic explanation. As such, even $\lambda=0$ can concur with the finite penetration length.

The models above show that the penetration length through a barrier and the phase shift are not tightly related and any value of $\lambda$ in Eq. (2) is admissible provided the existence of a short-ranged potential interpolating between the crystal bulk and the vacuum.
[1] M. Noguchi, K. Hirakawa, and T. Ikoma, Phys. Rev. Lett. 66, 2243 (1991).

[2] L. O. Olsson, C. B. M. Andersson, M. C. Håkansson, J. Kanski, L. Ilver, and U. O. Karlsson, Phys. Rev. Lett. 76, 3626 (1996).

[3] P. D. C. King, T. D. Veal, C. F. McConville, J. Zúñiga-Pérez, V. Muñoz-Sanjosé, M. Hopkinson, E. D. L. Rienks, M. F. Jensen, and P. Hofmann, Phys. Rev. Lett. 104, 256803 (2010).

[4] L. Colakerol, T. D. Veal, H.-K. Jeong, L. Plucinski, A. DeMasi, T. Learmonth, P.-A. Glans, S. Wang, Y. Zhang, L. F. J. Piper, P. H. Jefferson, A. Fedorov, T.-C. Chen, T. D. Moustakas, C. F. McConville, and K. E. Smith, Phys. Rev. Lett. 97, 237601 (2006).
[5] L. F. J. Piper, L. Colakerol, P. D. C. King, A. Schleife, J. ZunigaPerez, P.-A. Glans, T. Learmonth, A. Federov, T. D. Veal, F. Fuchs, V. Munoz-Sanjose, F. Bechstedt, C. F. McConville, and K. E. Smith, Phys. Rev. B 78, 165127 (2008).

[6] K. H. L. Zhang, R. G. Egdell, F. Offi, S. Iacobucci, L. Petaccia, S. Gorovikov, and P. D. C. King, Phys. Rev. Lett. 110, 056803 (2013).

[7] W. Meewasana, P. D. C. King, R. H. He, S.-K. Mo, M. Hashimoto, A. Tamai, P. Songsiriritthigul, F. Baumberger, and Z.-X. Shen, Nat. Mater. 10, 114 (2011).

[8] L. Colakerol, L. F. J. Piper, A. Fedorov, T. Chen, T. D. Moustakas, and K. E. Smith, Surf. Sci. 632, 154 (2015). 
[9] V. Yu. Aristov, G. Le Lay, V. M. Zhilin, G. Indlekofer, C. Grupp, A. Taleb-Ibrahimi, and P. Soukiassian, Phys. Rev. B 60, 7752 (1999).

[10] M. G. Betti, V. Corradini, G. Bertoni, P. Casarini, C. Mariani, and A. Abramo, Phys. Rev. B 63, 155315 (2001).

[11] M. Kobayashi, K. Yoshimatsu, E. Sakai, M. Kitamura, K. Horiba, A. Fujimori, and H. Kumigashira, Phys. Rev. Lett. 115, 076801 (2015).

[12] S. McKeown Walker, F. Y. Bruno, Z. Wang, A. de la Torre, S. Rico, A. Tamai, T. K. Kim, M. Hoesch, M. Shi, M. S. Bahramy, P. D. King, and F. Baumberger, Adv. Mater. 27, 3894 (2015).

[13] A. F. Santander-Syro, F. Fortuna, C. Bareille, T. C. Rodel, G. Landolt, N. C. Plumb, J. H. Dil, and M. Radovic, Nat. Mater. 13, 1085 (2014).

[14] A. B. Fowler, F. F. Fang, W. E. Howard, and P. J. Stiles, Phys. Rev. Lett. 16, 901 (1966), this example concerns 2DEG formed due to external electric field perpendicular to the surface.

[15] Y. J. Chang, L. Moreschini, A. Bostwick, G. A. Gaines, Y. S. Kim, A. L. Walter, B. Freelon, A. Tebano, K. Horn, and E. Rotenberg, Phys. Rev. Lett. 111, 126401 (2013).

[16] T. Ando, A. B. Fowler, and F. Stern, Rev. Mod. Phys. 54, 437 (1982).

[17] A. Ohtomo and H. Y. Hwang, Nature (London) 427, 423 (2004).

[18] Y. Chen, F. Trier, T. Kasama, D. V. Christensen, N. Bovet, Z. I. Balogh, H. Li, K. T. S. Thyden, W. Zhang, S. Yazdi, P. Norby, N. Pryds, and S. Linderoth, Nano Lett. 15, 1849 (2015).

[19] C. Cancellieri, A. S. Mishchenko, U. Aschauer, A. Filippetti, C. Faber, O. S. Barisic, V. A. Rogalev, T. Schmitt, N. Nagaosa, and V. N. Strocov, Nat. Commun. 7, 10386 (2016).

[20] W. Schmickler and E. Santos, in Interfacial Electrochemistry (Springer, Berlin, 2010), pp. 117-131.

[21] C. B. Duke, Phys. Rev. 159, 632 (1967).

[22] J. A. Appelbaum and G. A. Baraff, Phys. Rev. B 4, 1235 (1971).

[23] G. A. Baraff and J. A. Appelbaum, Phys. Rev. B 5, 475 (1972).

[24] X. Gao, E. Nielsen, R. P. Muller, R. W. Young, A. G. Salinger, N. C. Bishop, M. Lilly, and M. S. Carroll, J. Appl. Phys. 114, 164302 (2013).

[25] G. Paasch and H. Ubensee, Phys. Status Solidi B 113, 165 (1982).

[26] H. Ubensee, G. Paasch, J.-P. Zollner, and S. Handschack, Phys. Status Solidi B 147, 823 (1988).

[27] H. Ubensee, G. Paasch, and J. P. Zollner, Phys. Rev. B 39, 1955 (1989).

[28] P. D. C. King, T. D. Veal, and C. F. McConville, Phys. Rev. B 77, 125305 (2008).

[29] D. H. Ehlers and D. L. Mills, Phys. Rev. B 34, 3939 (1986).

[30] M. Lowe, T. D. Veal, A. P. Mowbray, and C. F. McConville, Surf. Sci. 544, 320 (2003).

[31] J. R. Weber, A. Janotti, and C. G. Van de Walle, Appl. Phys. Lett. 97, 192106 (2010).

[32] N. Tomaszewska, L. Walczak, J. Lis, and J. J. Kolodziej, Surf. Sci. 632, 103 (2015).

[33] R. Rehm, M. Masur, J. Schmitz, V. Daumer, J. Niemasz, T. Vandervelde, D. DeMeo, W. Luppold, M. Wauro, A. Wörl, F. Rutz, R. Scheibner, J. Ziegler, and M. Walther, Infrared Phys. Echnol. 59, 6 (2013).

[34] E.-Y. Chang, C.-I. Kuo, H.-T. Hsu, C.-Y. Chiang and Y. Miyamoto, Appl. Phys. Express 6, 034001 (2013).

[35] H. Yamaguchi, R. Dreyfus, S. Miyashita, and Y. Hirayama, Jpn. J. Appl. Phys. 41, 2519 (2002).
[36] V. Yu. Aristov, G. Le Lay, P. Soukiassian, K. Hricovini, J. E. Bonnet, J. Osvald, and O. Olsson, J. Vac. Sci. Technol. 12, 2709 (1994).

[37] M. G. Betti, R. Biagi, U. del Pennino, and C. Mariani, Europhys. Lett. 32, 235 (1995).

[38] V. Yu. Aristov, G. Le L. Lay, Thanh Vinh, K. Hricovini, and J. E. Bonnet, Phys. Rev. B 47, 2138 (1993).

[39] M. Lowe, T. D. Veal, C. F. McConville, G. R. Bell, S. Tsukamoto, and N. Koguchi, Surf. Sci. 523, 179 (2003).

[40] M. Lowe, T. D. Veal, C. F. McConville, G. R. Bell, S. Tsukamoto, and N. Koguchi, J. Cryst. Grow. 237, 196 (2002).

[41] The sulfur treatment allows us to keep ARPES images of 2DEG bands notably clear. The sulfur atoms are included in surface reconstructions to form a uniform (ordered) distribution of electrically active centers. This is in contrast to the case of adsorbed alkali atoms (forming chains and islands on the surface), which cause spatially nonuniform band bending and a distorted subband structure. Moreover, the surface disorder has a detrimental effect on ARPES spectra via scattering photoelectrons in their final states.

[42] G. R. Bell, C. F. McConville, and T. S. Jones, Phys. Rev. B 54, 2654 (1996).

[43] N. Olszowska and J. J. Kolodziej, Surf. Sci. 644, 95 (2016).

[44] W. Heegemann, K. H. Meister, E. Bechtold, and K. Hayek, Surf. Sci. 49, 161 (1975).

[45] K. A. Prior, J. M. Wallace, J. J. Hunter, S. J. A. Adams, M. J. L. S. Haines, M. Saoudi, and B. C. Cavenett, J. Cryst. Growth. 101, 176 (1990).

[46] S. Tsukamoto, T. Ohno, and N. Koguchi, J. Cryst. Growth. 176, 1303 (1997).

[47] Y. Fukuda, Y. Suzuki, N. Sanada, M. Shimomura, and S. Masuda, Phys. Rev. B 56, 1084 (1997).

[48] M. Katayama, M. Aono, H. Oigawa, Y. Nannichi, H. Sugahara, and M. Oshima, Jpn. J. Appl. Phys. 30, L786 (1991).

[49] E. Schillmann, in Compound Semiconductors, edited by R. K. Willardson and H. L. Goering (Reinhold, New York, 1962), Vol. 1, p. 358.

[50] H. Kato, M. Yokozawa, R. Kohara, Y. Okabayashi, and S. Takayanagi, Solid State Electron. 12, 137 (1969).

[51] Z. M. Fang, K. Y. Ma, D. H. Jaw, R. M. Cohen, and G. B. Stringfellow, J. Appl. Phys. 67, 7034 (1990).

[52] M. Grundmann, The Physics of Semiconductors (Springer, Berlin, 2006), p. 161.

[53] Clear 2DEG subbands images have been obtained for $\mathrm{CdO}$, in Refs. [5] and [3]. For both experiments the $\Delta$ value is about $0.2 \mathrm{eV}$ and its uncertainty must be also substantial. The case of InN in Ref. [8] is not interpretable because of undefined doping levels due to ion beam annealing treatment. For $\operatorname{As}(111)$, InN, $\mathrm{In}_{2} \mathrm{O}_{3}$, and $\mathrm{SrTiO}_{3}$ the obtained 2DEG structure images contain "pockets" filled with a signal in addition to (or even instead of) regular subbands.

[54] J. R. Chelikowsky and M. L. Cohen, Phys. Rev. B 14, 556 (1976).

[55] L. Walczak, G. Goryl, M. A. Valbuena, I. Vobornik, A. Tejeda, A. Taleb-Ibrahimi, J. J. Kolodziej, P. Segovia, and E. G. Michel, Surf. Sci. 608, 22 (2013).

[56] H. T. Luo, W. Z. Shen, Y. H. Zhang, and H. F. Yang, Physica B 324, 379 (2002).

[57] V. Palankovski, G. Kaibliger-Grujin, and S. Selberherr, Mater. Sci. Eng. B 66, 46 (1999). 
[58] S. C. Jain, J. M. McGregor, and D. J. Roulston, J. Appl. Phys. 68, 3747 (1990).

[59] Band gap reductions (BGN) due to the many-body interactions in electron-hole plasmas on weakly polar systems, for interparticle distance $\left(r_{s}\right)$ relevant to the case discussed here (i.e., $r_{s}$ within the range $0.1-0.5$ when expressed in effective Bohr radius units) may be (rather roughly) extrapolated based on the results shown in the paper by G. Tränkle, H. Leier, A. Forchel, H. Haug, C. Ell, and G. Weimann, Phys. Rev. Lett. 58, 419 (1987). The result is several $\mathrm{meV}$ for the $3 \mathrm{D}$ case and $<50 \mathrm{meV}$ for the strict $2 \mathrm{D}$ case. Similar values $(<50 \mathrm{meV}$ for the $2 \mathrm{D}$ case) may be estimated based on the work by Das Sarma, Jalabert, and Yang [Phys. Rev. B 39, 5516(R) (1989)]. This work, however, indicates also that for the quasi $2 \mathrm{D}$ case (what is our case) the $\mathrm{BGN}$ is reduced as compared to the strict $2 \mathrm{D}$ case. This reduction is very significant already for the plasma layer thickness corresponding to the 0.25 Bohr radius. While both papers above are not strictly relevant, since they concern the electron-hole plasmas, nevertheless the estimations of the relative 2D/3D BGN should be applicable.

[60] E. F. Schubert, Doping in III-V Semiconductors (Cambridge University Press, Cambridge, 1993), Chap. 3.1.

[61] G. Bonneau, J. Faraut, and G. Valent, Am. J. Phys. 69, 322 (2001).

[62] S. Abe, T. Inaoka, and M. Hasegawa, Phys. Rev. B 66, 205309 (2002).

[63] M. Klaus and B. Simon, Ann. Phys. 130, 251 (1980).

[64] W. G. Kelley and A. C. Peterson, The Theory of Differential Equations (Springer, New York, 2010), Chap. 8.7. 